\title{
On Equality and Strong Equality of Domination Number and Independent Domination Number in Graphs
}

Femlin Pious* and Mayamma Joseph ${ }^{\dagger}$

\section{Abstract}

In this paper we explore graphs having same domination number $\gamma$ and independent domination number $\gamma_{i}$. Such graphs are denoted as $\left(\gamma, \gamma_{i}\right)$-graphs. Several families of $\left(\gamma, \gamma_{i}\right)$-graphs have been constructed. The realization problem for graphs with $\gamma=\gamma_{i}=a$ for any given positive integer $a$ has been solved. Furthermore, properties of graphs in which every $\gamma$-set is a $\gamma_{i}$-set has been investigated.

Keywords: Domination, Independent domination, Efficient dominating set, Strong equality of domination parameters.

\section{Introduction}

Let graph $G=(V, E)$ be a simple finite connected graph of order $n$. The open neighborhood of a vertex $\mathrm{v} \in \mathrm{V}$ is $\mathrm{N}(\mathrm{v})=\{\mathrm{u} \in \mathrm{V} \mid \mathrm{vu} \in \mathrm{E}\}$. The closed neighborhood is $\mathrm{N}[\mathrm{v}]=\mathrm{N}(\mathrm{v}) \cup\{\mathrm{v}\}$. A corona $\mathrm{G} \circ K_{1}$ of a graph $\mathrm{G}$ is the graph obtained from $\mathrm{G}$ by adding a new vertex $\mathrm{u}$ and

* Department of Mathematics, Christ University, Bangalore-560029, India; femlin.pious@gmail.com

† Department of Mathematics, Christ University, Bangalore-560029, India; mayamma.joseph@christuniversity.in 
the pendent edge uv for every vertex $\mathrm{v} \in \mathrm{V}(\mathrm{G})$. Subdivision is the operation of replacing an edge by a path of two edges through a new vertex. A graph obtained from a graph $\mathrm{H}$ by successive edge subdivisions is called $H$-subdivision (or subdivision of $H$ ).

A vertex of degree one is called a leaf. A support vertex is a vertex that is adjacent to a leaf, while a strong support vertex is adjacent to at least two leaves. A tree with at most two vertices of degree more than one is called a double star.

A set $\mathrm{S}$ of vertices is a dominating set if every vertex in $\mathrm{V}$ is either in $\mathrm{S}$ or adjacent to a vertex in $\mathrm{S}$. The minimum cardinality of a dominating set is the domination number $\gamma(\mathrm{G})$. A dominating set of cardinality $\gamma(\mathrm{G})$ is called $\gamma$-set of $\mathrm{G}$ (or simply $\gamma(\mathrm{G})$-set) .

The fascinating fact is that we can define various types of domination parameters by imposing several graph theoretic properties in the definition of dominating set. A dominating set is an independent dominating set if it is independent. An independent domination number $\gamma_{i}$ is the minimum cardinality of an independent dominating set. We call a minimum independent dominating set of a graph $\mathrm{G}$ a $\gamma_{i}$-set of $\mathrm{G}$ or $\gamma_{i}(\mathrm{G})$-set.

Since every independent dominating set is a dominating set, for any graph $\mathrm{G}, \gamma(\mathrm{G}) \leq \gamma_{i}(\mathrm{G})$. There are graphs for which independent domination number is same as the domination number. However, it is not necessary that every $\gamma$-set will be a $\gamma_{i}$ set for a graph with $\gamma=\gamma_{i}$. First we explore graphs whose independent domination number is the same as its domination number.

\section{2. $\left(\gamma, \gamma_{i}\right)$-Graphs}

We call graphs $\mathrm{G}$ which satisfies the relation $\gamma(\mathrm{G})=\gamma_{i}(\mathrm{G})$ as $\left(\gamma, \gamma_{i}\right.$ ) -graphs. The complete graph $K_{n}$, star graph $K_{1, t}$, wheel graph $W_{n}$ , path graph $P_{n}$ and cycle graph $C_{n}$ are some of the standard classes of $\left(\gamma, \gamma_{i}\right)$ - graphs. 
Theorem 1. If $\mathrm{G}$ is a graph with $\gamma_{i}(\mathrm{G}) \leq 2$, then $\mathrm{G}$ is a $\left(\gamma, \gamma_{i}\right)$-graph.

Proof. We have $1 \leq \gamma(\mathrm{H}) \leq \gamma_{i}(\mathrm{H})$ for every graph $\mathrm{H}$. It follows that if $\mathrm{G}$ is a graph with $\gamma_{i}(\mathrm{G})=1$, then $\gamma(\mathrm{G})=\gamma_{i}(\mathrm{G})$. Suppose $\mathrm{G}$ be a graph with $\gamma_{i}(\mathrm{G})=2$. If possible, let $\gamma(\mathrm{G})=1$, then since every singleton set is an independent set, $\gamma_{i}(G)=1$, a contradiction. Therefore, if $G$ is a graph with $\gamma_{i}(G)=2$, then $\gamma(G)=\gamma_{i}(G)$. Hence, if $\mathrm{G}$ is a graph with $\gamma_{i}(\mathrm{G}) \leq 2$, then $\mathrm{G}$ is a $\left(\gamma, \gamma_{i}\right)$-graph.

If a graph $G$ has a set $S$ such that the collection of closed neighborhoods of vertices of $S$ gives a partition, then $S$ is definitely a dominating set of $G$. Bange et al. [1] have named such a set $S$ such that $\{\mathrm{N}[\mathrm{s}]: \mathrm{s} \in \mathrm{S}\}$ is a partition as Efficient Dominating Set(or EDS). Goddard and Henning [6] have shown that a graph having an EDS is a $\left(\gamma, \gamma_{i}\right)$-graphs. Note that it is not compulsory for a graph to have an EDS. For instance, there is no set $\mathrm{X} \subseteq V\left(C_{4}\right)$ such that collection of closed neighborhoods of vertices of $\mathrm{X}$ forms a partition in cycle $C_{4}$.

If $S$ is an EDS of a graph $G$, then the closed neighborhoods of vertices of $S$ are disjoint sets. This implies that $S$ is a set of nonadjacent vertices. Therefore, the following remark is quite obvious.

Remark 1. An efficient dominating set of a graph $\mathrm{G}$ is independent.

The converse of Remark $\mathbf{1}$ is not true always. Every EDS is a dominating set, but keep in mind that every $\gamma(\mathrm{G})$ - set need not be an EDS. We can observe that an independent set of $C_{4}$ is not an EDS and no $\gamma\left(C_{4}\right)$-set is an EDS.

\section{Construction of families of $\left(\gamma, \gamma_{i}\right)$-graphs}

In this section, we attempt to construct some families of graphs having equal domination number and independent domination number. 


\subsubsection{Dumbbell graphs $D_{r, s}$}

Consider two complete graphs $K_{r}$ and $K_{s}$ on $\mathrm{r}$ and s vertices, respectively. Connect $K_{r}$ and $K_{s}$ by an edge e. Thus we obtain a simple graph called Dumbbell graph, denoted by $D_{r, s}$.

Proposition 1.Dumbbell graph $D_{r, s}$ is a $\left(\gamma, \gamma_{i}\right)$-graph.

Proof. We claim that $\gamma\left(D_{r, s}\right)=\gamma_{i}\left(D_{r, s}\right)$. Let $\mathrm{u} \in \mathrm{V}\left(K_{r}\right)$ and $\mathrm{v} \in \mathrm{V}($ $\left.K_{s}\right)$. Then the set $\{\mathrm{u}, \mathrm{v}\}$ is a dominating set of $D_{r, s}$ for any vertices $\mathrm{u}$ and v. So, $\gamma\left(D_{r, s}\right) \leq 2$. Further, there is no vertex of degree $(\mathrm{r}+\mathrm{s}$ - 1), showing that $\gamma\left(D_{r, s}\right)=2$. Moreover, the set $\{\mathrm{u}, \mathrm{v}\}$ is an independent dominating set for all non-adjacent pair of vertices $\mathrm{u}$ and $\mathrm{v}$ such that $\mathrm{u} \in \mathrm{V}\left(K_{r}\right)$ and $\mathrm{v} \in \mathrm{V}\left(K_{s}\right)$. Hence, $\gamma_{i}\left(D_{r, s}\right) \leq 2$. Since every independent dominating set is a dominating set we conclude that $\gamma_{i}\left(D_{r, s}\right)=2$.

Figure 1 depicts Dumbbell graph $D_{3,4}$ obtained by connecting complete graphs $K_{3}$ and $K_{4}$ by an edge.

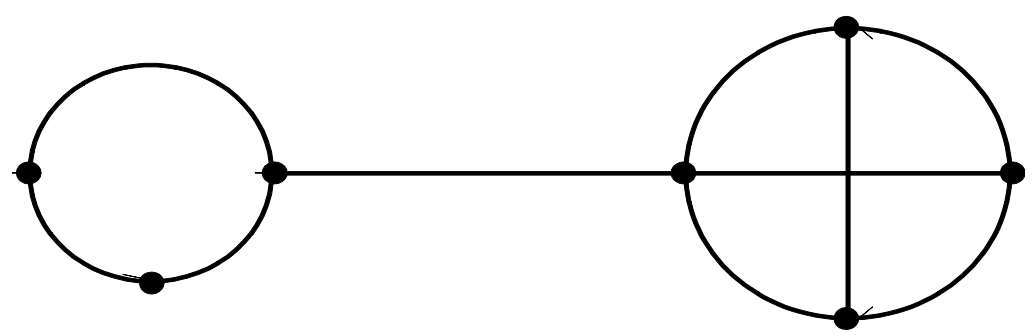

Fig 1. $\gamma^{\gamma}\left(D_{3,4}\right)=\gamma_{i}\left(D_{3,4}\right)=2$

Now we proceed to construct another family of $\left(\gamma, \gamma_{i}\right)$-graphs. 


\subsubsection{Sunrise graphs $S_{r}$}

Consider a complete graph $K_{r}$ and r- copies of star $K_{1,2}$. Connect a leaf of $K_{1,2}$ to each vertex of $K_{r}$ using an edge. Thus we obtain a simple graph and we call it a Sunrise graph, denoted by $S_{r}$.

Figure 2 shows Sunrise graph $S_{3}$. It is obtained by connecting a leaf of $K_{1,2}$ to each vertex of complete graph $K_{3}$ by an edge.

Since no vertex of $S_{r}$ has three pair-wise non-adjacent neighbors, sunrise graphs are claw-free. Therefore, $S_{r}$ is a $\left(\gamma, \gamma_{i}\right)$-graph as every claw-free graphs are $\left(\gamma, \gamma_{i}\right)$-graphs $[5,6]$.

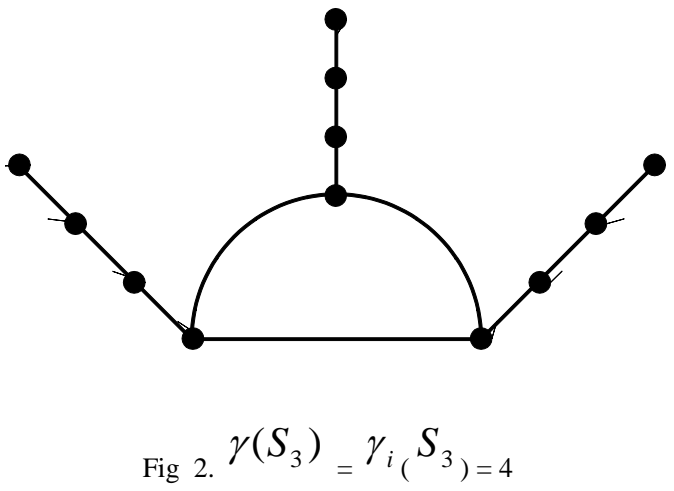

Let $\mathrm{z}$ be a vertex of complete graph $K_{r}$ and $\mathrm{x}_{1}, \mathrm{x}_{2}, \ldots, \mathrm{x}_{\mathrm{r}}$ be the central vertices of stars in sunrise graph $S_{r}$. Then $\left\{\mathrm{z}_{,} \mathrm{x}_{\mathrm{j}} \mid 1 \leq \mathrm{j} \leq \mathrm{r}\right\}$ is the $\gamma\left(S_{r}\right)$-set. In addition, these $\mathrm{r}+1$ non-adjacent vertices are required to dominate the graph. Therefore, $\gamma\left(S_{r}\right)=\gamma_{i}\left(S_{r}\right)=\mathrm{r}+1$. Hence we have the following proposition.

Proposition 2. Let $S_{r}$ be a sunrise graph. Then $\gamma\left(S_{r}\right)=\gamma_{i}\left(S_{r}\right)=\mathrm{r}$ +1 .

The above proposition also leads us to the realization problem of constructing a graph $\mathrm{G}$ such that $\gamma(\mathrm{G})=\gamma_{i}(\mathrm{G})=\mathrm{k}$ for any positive integer ' $\mathrm{k}$ '. This is achieved by considering the sunrise graph $S_{k-1}$. 
Theorem 2. Given any positive integer ' $k$ ', there exists a graph $\mathrm{G}$ with $\gamma(\mathrm{G})=\gamma_{i}(\mathrm{G})=k$.

\section{Strong equality between $\gamma$ and $\gamma_{i}$}

This section deals with the concept called strong equality of domination number and independent domination number in graphs. This concept of strong equality of two graph theoretic parameters was introduced by Haynes et al. [2, 4].

Let $P_{1}$ and $P_{2}$ be properties of vertex subsets of a graph $\mathrm{G}$, and assume that every subset of $\mathrm{V}(\mathrm{G})$ with property $P_{2}$ also has property $P_{1}$. Let $\psi_{1}(\mathrm{G})$ and $\psi_{2}(\mathrm{G})$ denote the minimum cardinalities of sets with properties $P_{1}$ and $P_{2}$ respectively. Then $\psi_{1}(\mathrm{G}) \leq \psi_{2}(\mathrm{G})$. If $\psi_{1}(\mathrm{G})=\psi_{2}(\mathrm{G})$ and every $\psi_{1}(\mathrm{G})$-set is also a $\psi_{2}(\mathrm{G})$-set, then we say that $\psi_{1}(\mathrm{G})$ strongly equals $\psi_{2}(\mathrm{G})$, written $\psi_{1}(G) \equiv \psi_{2}(G)$. It is shown that for a path $P_{n}$ and cycle $C_{n}$

$$
\begin{aligned}
& \gamma_{i}\left(C_{3 k}\right) \equiv \gamma\left(C_{3 k}\right)=\gamma_{i}\left(P_{3 k}\right) \equiv \gamma\left(P_{3 k}\right)=k \\
& \gamma_{i}\left(C_{3 k+2}\right) \equiv \gamma\left(C_{3 k+2}\right)=\gamma_{i}\left(P_{3 k+2}\right) \equiv \gamma\left(P_{3 k+2}\right)=k+1
\end{aligned}
$$

Note that $\gamma$ and $\gamma_{i}$ are not strongly equal for path $P_{3 k+1}$ and cycl $C_{3 k+1}$ even though $\gamma_{i}\left(C_{3 k+1}\right)=\gamma\left(C_{3 k+1}\right)=\gamma_{i}\left(P_{3 k+1}\right)=\gamma\left(P_{3 k+1}\right)=k+1$ , where $\mathrm{k}$ is any positive integer [2].

If a graph $\mathrm{G}$ satisfies the relation $\gamma=\gamma_{i}$ and every $\gamma$-set of $\mathrm{G}$ is also a $\gamma_{i}(G)$-set, then $\gamma(G)$ strongly equals $\gamma_{i}(G)$, denoted as $\gamma(G) \equiv \gamma_{i}(G)$. We know that $\gamma\left(P_{5}\right)=\gamma_{i}\left(P_{5}\right)=2$ and each of the three $\gamma\left(\mathrm{P}_{5}\right)$-sets is also a $\gamma_{i}$-set of $P_{5}$. Therefore $\gamma\left(P_{5}\right) \equiv \gamma_{i}\left(P_{5}\right)$ .Whereas in cycle $C_{4}$ which also is a $\left(\gamma, \gamma_{i}\right)$-graph, we can see that not every $\gamma$-set is a $\gamma_{i}$-set and so $\gamma\left(C_{4}\right)$ and $\gamma_{i}\left(C_{4}\right)$ are not strongly equal. 
3.1 Construction of families admitting strong equality between $\gamma$ and $\gamma_{i}$

It is to be noted that $\gamma$ and $\gamma_{i}$ are strongly equal for path $P_{n}$ and cycle $C_{n}$ if $\mathrm{n}=3 \mathrm{k}$ and $\mathrm{n}=3 \mathrm{k}+2[2,3,4]$.

For the tree in Figure 3, the sets $\left\{z_{i} \mid 1 \leq \mathrm{i} \leq \mathrm{t}\right\} \cup\{\mathrm{w}\}$ and $\left\{\mathrm{y}_{\mathrm{i}} \mid 1 \leq \mathrm{i}\right.$ $\leq \mathrm{t}\} \cup\{\mathrm{w}\}$ are the $\gamma$ sets and moreover, they are independent sets. Thus $\gamma_{i}=\gamma=\mathrm{t}+1$. Since every $\gamma$-set is a $\gamma_{i}$-set, $\gamma$ strongly equals $\gamma_{i}$.

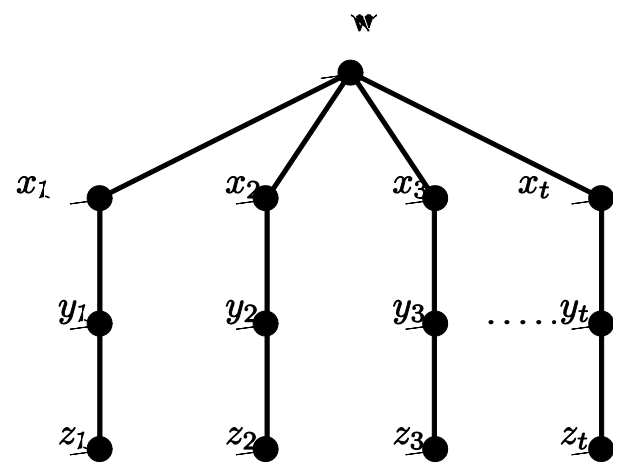

Fig 3. $\gamma_{i} \equiv \gamma=\mathrm{t}+1$

The set $\left\{b_{i}, p_{i} \mid 1 \leq \mathrm{i} \leq \mathrm{t}\right\}$ is the $\gamma$-set of the tree in Figure 4. As the same set is independent too, its domination number and independent domination number is equal to $2 t$. Therefore, it admits strong equality between $\gamma$ and $\gamma_{i}$.

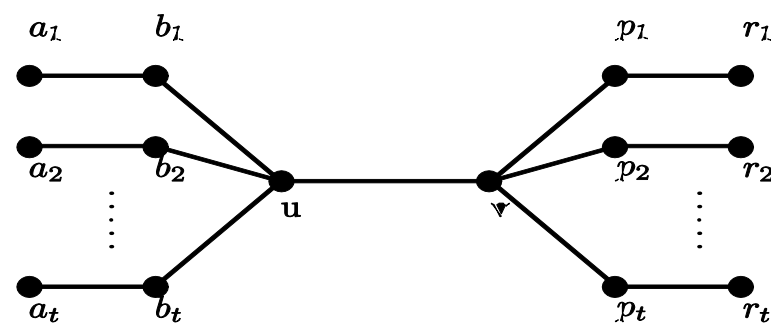

Fig 4. $\gamma_{i} \equiv \gamma=2 \mathrm{t}$ 
Recall that paths and cycles are claw-free. But the path $P_{3 k+1}$ and cycle $C_{3 k+1}$ never admits strong equality between $\gamma$ and $\gamma_{i}$.

The following observations provide few families of graphs which never possess strong equality.

Observation 1. Every claw-free graphs need not have strong equality between $\gamma$ and $\gamma_{i}$.

Observation 2. The corona of graphs never admit strong equality between domination number and independent domination number.

Our aim is to construct a family of graphs admitting strong equality between $\gamma$ and $\gamma_{i}$. For the purpose of construction, consider a complete graph $K_{q}$ on q vertices and 'q' number of stars $K_{1,(q-1)}$. Then, connect an edge from the central vertex of a star to a vertex of $K_{q}$. The resulting graph is a simple graph and we wish to call it as Umbel graph, $U_{q}$ as it bears some resemblance of an inflorescence called Umbel.

The Figure 5 shows Umbel graph $U_{4}$

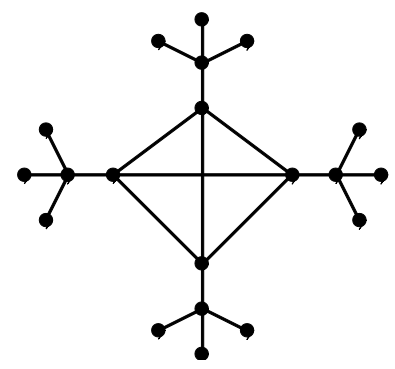

Fig 5. $\gamma\left(U_{4}\right)=\gamma_{i}\left(U_{4}\right)=4$

This family of graphs can be extended to a wider class. Consider a complete graph $K_{q}$ on q vertices and $\left|\mathrm{V}\left(K_{q}\right)\right|$ number of stars on vertices $t_{1}, t_{2}, \ldots, t_{q}$ where $t_{i}, 1 \leq \mathrm{i} \leq \mathrm{q}$ is an integer. Next, connect 
an edge from the center vertex of a star to a vertex of $K_{q}$. We can observe that the family of resulting graphs is an extension of family of umbel graphs. These graphs admit strong equality between $\gamma$ and $\gamma_{i}$ as the only $\gamma$-set is the set of central vertices of all stars and moreover, it is an independent set.

Hence, we have the following theorem.

Theorem 3. Given any positive integer q, there exists a graph $\mathrm{G}$ such that $\gamma(G) \equiv \gamma_{i}(G)=\mathrm{q}$.

\section{Conclusion}

In this paper we have discussed several families of $\left(\gamma, \gamma_{i}\right)$-graphs. New families of such graphs have been constructed. We analyzed and discussed the concept of strong equality of domination parameters. Particularly, we focused on strong equality between $\gamma$ and $\gamma_{i}$. New families of graphs having strong equality between $\gamma$ and $\gamma_{i}$ have been constructed. We proved that there exists a graph such that $\gamma(G) \equiv \gamma_{i}(G)=\mathrm{q}$.

Studies could be extended to obtain more families of graphs with $\gamma \equiv \gamma_{i}$ and characterization of such graphs.

\section{References}

[1] D. W. Bange , A. E. Barkauskas, L. H. Host and P.J. Slater, "Generalized domination and efficient domination in graphs," Discrete Math., vol. 159, no. 1, pp. 1-11, 1996.

[2] T. W. Haynes, M. A. Henning and P. J. Slater, "Strong equality of domination parameters in trees," Discrete Math., vol. 206, no. 1-3, pp. 77-87, 2003.

[3] T. W. Haynes and M. A. Henning, "Trees with large paireddomination number," Util. Math., vol. 71, no. 1, pp. 3-12, 2006.

[4] T. W. Haynes and P. J. Slater, "Paired-domination in graphs, DiscreteMath., vol. 32, no. 3, pp. 199-206, 1998. 
[5] W. Goddard and M. A. Henning, J. Lyle and J. Southey, "On the independent domination in graphs", Ann. Comb., vol. 16, no. 4, pp. 719-732, 2012.

[6] W. Goddard and M. A. Henning, "Independent domination in graphs: a survey and recent results", Discrete Math., vol. 313, no. 7, pp. 839-854, 2013. 